\title{
Henoch Schonlein Purpura as Possible Sole Manifestation of Covid-19 in Children
}

\section{Sarah Falou ${ }^{1 *}$, Ghina Kahil ${ }^{2}$, Bassem Abou Merhi ${ }^{1}$, Rouwayda Dana ${ }^{1}$ and Imad Chokr ${ }^{1}$}

${ }^{1}$ Department of Pediatrics, Lebanese University, Faculty of Medical Sciences,

Lebanon

${ }^{2}$ Depatment of Pediatrics, Beirut Arab University, Faculty of Medical Sciences,

Lebanon

*Corresponding Author: Sarah Falou, Department of Pediatrics, Lebanese

University, Faculty of Medical Sciences, Beirut, Lebanon.
Received: February 19, 2021

Published: March 11, 2021

(C) All rights are reserved by Sarah Falou., et al.

\begin{abstract}
Coronavirus disease-19 (Covid-19), which was isolated for the first time in Wuhan China, in December 2019 is an ongoing global pandemic caused by the SARS-CoV-2, a single-stranded RNA virus from the Beta-Coronaviridae family. Common presentation includes respiratory symptoms ranging from a mild common cold-like illness to severe pneumonia. However, covid-19 infection exhibits also extrapulmonary manifestations, including cardiac, dermatological, thrombotic and hepatocellular symptoms that have been increasingly reported in the last few months. Purpuric lesions have been suggested to occur more frequently in elderly patients with severe Covid-19, likely representing the cutaneous manifestations associated with the highest rate of Covid-19 related mortality. Besides, Henoch-Schonlein Purpura (HSP) is the most common form of vasculitis in children that affects several systems. It can occur secondary to upper respiratory tract infections, medications, vaccinations and malignancies. We describe herein, a case of an 8-yearold boy who presented with inability to bear weight and purpura, clinically diagnosed as Henoch-Schonlein Purpura vasculitis (HSP) with concurrent Covid-19 infection confirmed by positive PCR on nasopharyngeal sample. This case highlights a newly described presentation of Covid-19 infection.
\end{abstract}

Keywords: COVID-19; Henoch-Schönlein Purpura (HSP); Arthralgia; Inability to Bear Weight

\section{Abbreviations}

PCR: Polymerase Chain Reaction; SARS-CoV-2: Severe Acute Respiratory Syndrome Coronavirus 2; RNA: Ribonucleic Acid; $E U$ LAR: European League Against Rheumatism; PRINTO: Pediatric Rheumatology International Trials Organisation; PRES: Pediatric Rheumatology European Society; CRP: C-Reactive Protein; NSAIDs: Non-Steroidal Anti-Inflammatory Drugs; IgA: Immunoglobulin A

\section{Introduction}

Coronavirus disease 2019 (Covid-19) is caused by severe acute respiratory syndrome coronavirus 2 (SARS-CoV-2) first identified in December 2019. Since the start of the pandemic, fewer cases of Covid-19 have been reported in children and most were mild presenting with cough and fever. Although most children infected with (SARS-CoV-2) have either asymptomatic infection or mild symptoms, a severe illness has also been reported. Treatment of Covid-19 is mainly supportive [1]. Henoch-Schonlein purpura (HSP) is the most common vasculitis in children and is characterized by leukocytoclastic and immunoglobulin A deposition in the small vessels in the skin, joints, gastrointestinal tract, and kidneys. It usually occurs between ages 3 and 10 and affects males more frequently 
than females. The incidence is estimated to be $14-20$ per 100,000 children per year. The hallmark of HSP is its rash: palpable purpura that typically lasts 3 - 10 days. In $75 \%$ of the cases arthralgia and arthritis occur. The latter tends to be self-limited resolving within 2 weeks. Periarticular swelling and tenderness without erythema or effusions are common. In addition, in up to $80 \%$ of cases there's gastrointestinal (GI) involvement. Renal involvement occurs in up to $30 \%$ of cases and can be delayed for several months after the initial illness. The diagnosis of HSP is clinical; with normal platelet count on complete blood count. Assessment of renal involvement with blood pressure, urine analysis and serum creatinine is necessary. Treatment is generally supportive, and the prognosis is excellent [2].

\section{Case Presentation}

A previously healthy 8 years old male patient presented to ER for a new onset skin rash located on both lower limbs appearing 4 days prior to presentation. History goes back to one week prior to presentation, when the patient was diagnosed with Covid19 infection; being totally asymptomatic. His infection was discovered incidentally after doing PCR-Covid-19 test for being in contact with a family member infected with corona virus. Upon presentation, vitals were within normal limits, the patient was complaining of bilateral lower limb rash associated with bilateral ankle pain advancing to inability to bear weight or stand up. Patient denied fever, abdominal pain, and presence of urinary symptoms. On physical exam, no significant findings were found, except for ecchymosis and purpura on both lower limbs, in addition to bilateral ankle edema (Figure 1) along with decreased range of motion and tenderness.

The Patient was admitted for further management and investigation. Laboratory results showed: normal creatinine value $[0.37$ $\mathrm{mg} / \mathrm{dL}$ ), negative CRP (1.5 mg/L) and a normal platelets count 252 $x 10^{\wedge} 9 /$ L. and normal urine analysis. During his hospital stay, the patient was started on intravenous hydration along with symptomatic treatment including NSAIDs and paracetamol. Patient's urine output was also monitored and observed for any change in color, quantity and other characteristics. On the second day of hospitalization, a decrease in ankle edema and purpuric rash was observed with mild improvement in the range of motion in both ankles. On the fifth day of hospitalization, the patient regained his ability to stand up and bear his weight with almost complete resolution of rash. Patient was then discharged home with diagnosis of HSP associated with Covid-19 infection.

\section{Discussion}

Based on the EULAR/PRINTO/PRES criteria; a patient is classified as HSP in the presence of purpura or petechiae (mandatory) with lower limb predominance, plus one of four criteria: acute abdominal pain; histopathology showing leukocytoclasic vasculitis or proliferative glomerulonephritis with predominant IgA deposits; arthritis or arthralgia and renal involvement in the form of proteinuria or hematuria [3]. Since our patient had palpable purpura in the absence of thrombocytopenia and acute onset of arthralgia, he had the clinical signs and symptoms of HSP and was diagnosed accordingly as HSP. More than $90 \%$ of patients with HSP are below 10 years of age, with the respiratory illness being the most common preceding infection. The most common associated pathogens are Para influenza virus and Streptococcus strains, besides; Human Parvovirus B19 is a frequent viral trigger in children [4].

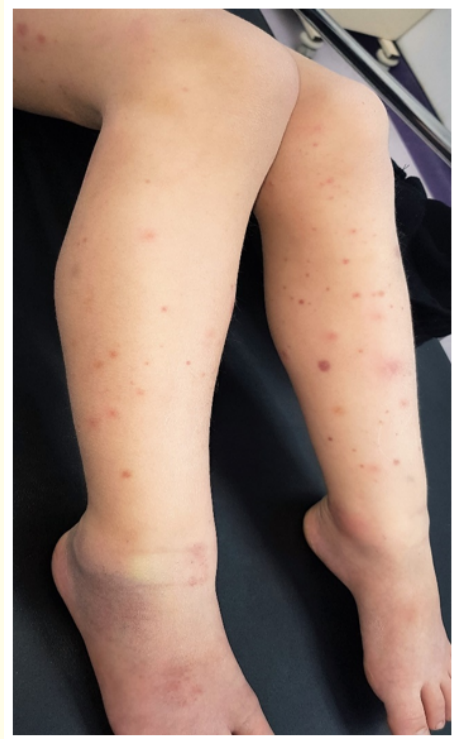

Figure 1: Showing ecchymosis and purpura on both lower limbs with bilateral ankle edema.

Our patient did not have any prior infection with the previously mentioned organisms. The fact of coexisting covid-19 infection suggests that Covid-19 organism could possibly be the virus that triggers HSP. To our knowledge, 2 cases were reported stating the occurrence of covid-19 infection and HSP in the literature, the first being in a 78 years old man who presented with arthritis, cutaneous vasculitis and nephritic syndrome following Covid-19 pneumo- 
nia where kidney biopsy was the confirming diagnostic procedure [5]. The second was a case of a 4 years old boy, who presented with a 1-day history of pruritic, non-blanching, maculopapular rash all over his lower limbs bilaterally, and who had recently recovered from an upper respiratory tract infection caused by Covid-19, exactly 37 days before presenting with his skin lesions [6]. Moreover, a review by Abdel Massih., et al. reported a link between Covid-19 and another childhood vasculitis, Kawasaki disease [7]. Another case reported by Chesser., et al. also stated a link between Covid-19 and acute hemorrhagic edema of infancy in an 8-month-old girl [8]. Concerning our patient, he presented with inability to stand on his feet on his own normally, "I can't bear my weight" he said, and these purpuric rashes with concurrent infection by covid-19 that was confirmed by PCR, and what makes our case distinguished, is the fact that these skin lesions were the only presenting signs upon a confirmed covid-19 infection without any respiratory symptoms. Alternatively, more research is necessary to determine the role of SARS-CoV-2 in the pathogenesis of IgA-mediated vasculitis, the frequent association between HSP and respiratory viruses, along with the Covid-19 infection in our patient and the previous cases cited suggest that there may be a link between Covid-19 infection and HSP and signals that HSP could be the presenting sign in Covid-19 infection.

\section{Conclusion}

We encountered and thus reporting a case of HSP in association with Covid-19 infection. SARS-CoV-2 IgA, being the first immunoglobulin to rise following Covid-19 infection [9], the possible association between IgA vasculitis and Covid-19 infection is proposed. With the continuous increase in Corona virus infection spread, it is likely for physicians to encounter more cases of Covid-19-associated with HSP, where HSP may be the only or first presenting sign. That makes sharing our case of high importance, for guidance, early diagnosis and management.

\section{Bibliography}

1. Cascella Marco., et al. "Features, evaluation and treatment coronavirus (COVID-19)". Statpearls (2020).

2. Kliegman RM and Geme JW. "Rheumatic Diseases of Childhood (Connective Tissue Disease, Collagen Vascular Diseases)". In Nelson textbook of pediatrics 2 .

3. Ozen Seza., et al. "EULAR/PRINTO/PRES criteria for HenochSchönlein purpura, childhood polyarteritis nodosa, childhood
Wegener granulomatosis and childhood Takayasu arteritis: Ankara 2008. Part II: Final classification criteria". Annals of the Rheumatic Diseases 69.5 (2010): 798-806.

4. Hetland Liv Eline., et al. "Henoch-Schönlein Purpura: A Literature Review”. Acta Dermato-Venereologica 97.10 (2017): 11601166.

5. Suso Andrea S., et al. "IgA Vasculitis With Nephritis (HenochSchönlein Purpura) in a COVID-19 Patient". Kidney International Reports 5.11 (2020): 2074-2078.

6. AlGhoozi Dalal Anwar and Haya Mohammed AlKhayyat. "A child with Henoch-Schonlein purpura secondary to a COVID-19 infection". BMJ Case Reports CP 14.1 (2021): e239910.

7. AbdelMassih Antoine Fakhry., et al. "Unleashing the mysterious link between COVID-19 and a famous childhood vasculitis: Kawasaki disease". Egyptian Pediatric Association Gazette 68.1 (2020): 21.

8. Chesser Hannah., et al. "Acute Hemorrhagic Edema of Infancy after Coronavirus Infection with Recurrent Rash". Case Reports in Pediatrics (2017): 5637503.

9. Jacobi Michal., et al. "Purpurona: A Novel Report of COVID19-Related Henoch-Schonlein Purpura in a Child". The Pediatric Infectious Disease Journal 40.2 (2021): e93-e94.

\section{Assets from publication with us}

- Prompt Acknowledgement after receiving the article

- Thorough Double blinded peer review

- Rapid Publication

- Issue of Publication Certificate

- High visibility of your Published work

Website: www.actascientific.com/

Submit Article: www.actascientific.com/submission.php

Email us: editor@actascientific.com

Contact us: +919182824667 